\title{
Celulite facial em lábio inferior oriunda de acne inflamada: relato de caso
}

\author{
Facial cellulitis in lower lip caused by inflamed acne: case report
}

\author{
Marcelo Bighetti Toniollo* \\ Andrea Sayuri Silveira Dias Terada* \\ Enzo Melchior Junior ${ }^{* * *}$
}

\section{Resumo}

Introdução: a celulite facial é a difusão de processo inflamatório por planos subcutâneos em tecidos moles, podendo ter sua etiologia oriunda de variados fatores, sendo, dentre eles, a origem odontogênica a mais comumente encontrada. Entretanto, a celulite facial pode também estar relacionada a causas menos comuns, como processos infecciosos de origem não odontogênica. Objetivo: o presente relato de caso vem a elucidar o início de um processo infeccioso gerado por acne inflamada na borda inferior do lábio, a qual foi a porta de entrada para bactérias, o que gerou a difusão do processo inflamatório para planos subcutâneos e originou uma celulite facial. Relato de caso: pelo fato de não haver agente causal específico, como as originadas por causa odontogênica, foi estabelecido tratamento com antibióticoterapia de amplo espectro, associado à meticulosa higienização e terapia com calor, a fim de induzir abscedação espontânea. Exames específicos laboratoriais também foram solicitados para maior segurança e diagnóstico preciso do profissional. A evolução do caso transcorreu em oito dias, de forma rápida e aguda, principalmente nos primeiros cinco dias. Considerações finais: conforme relatado no caso, a interação entre o cirurgião-dentista e o médico bem como a presença da interdisciplinaridade foram fatores fundamentais no tratamento como um todo e para a resolução do caso de forma satisfatória, uma vez que a ocorrência de celulite facial nessa área não é geralmente presenciada.

Palavras-chave: Celulite facial. Drenagem. Infecção. Lábio inferior.

\section{Introdução}

A celulite facial é um processo inflamatório agudo difuso nos tecidos subcutâneos. Trata-se de uma espécie de abscesso que não drena via extra ou intrabucal, o que gera aumento de volume pela sua difusão através dos planos faciais dos tecidos moles $^{1}$. Geralmente, é causada por bactérias do tipo estreptococos ou estafilococos, as quais se infiltram nas camadas subcutâneas por possíveis portas de entrada ${ }^{2}$. Quando tal infecção evolui e toma forma de Angina de Ludwig, Trombose do Seio Cavernoso, Mediastinite ou mesmo se aproxima das meninges e do cérebro, o paciente corre riscos mais elevados, podendo gerar septicemia e chegar à morte ${ }^{1,3,4}$.

É sabido que pacientes imunodeprimidos, alcoólatras e/ou diabéticos apresentam maior susceptibilidade a variadas doenças e infecções, inclusive à celulite facial e suas complicações, que tem maior incidência em crianças e adultos jovens ${ }^{5,6}$. Além disso, dependendo da área afetada pela celulite facial, os tecidos loco-regionais apresentam dilatação e extensão variadas, de acordo com a resistência muscular e do tecido ósseo ${ }^{7,8}$.

Variados são os casos de relato de celulite facial associados à origem odontogênica, causa mais comum $^{1,6}$. No entanto, tal inflamação pode advir de outras fontes, correlatas ou distintas, tais como: foco de infecção dentária, como cáries ou periodontites, infecção em procedimentos cirúrgicos, cortes e

\footnotetext{
Especialista em Prótese Dentária pela AORP. Mestre e doutor em Reabilitação Oral pela FORP-USP. Docente da Faculdade de Odontologia de Rio Verde, Universidade de Rio Verde (FORV/UniRV), Rio Verde, GO, Brasil.

- Doutoranda em Ciências da Saúde na Faculdade de Medicina de Ribeirão Preto, FMRP/USP. Docente da Faculdade de Odontologia de Rio Verde, Universidade de Rio Verde (FORV/UniRV), Rio Verde, GO, Brasil.

*** Dermatologista pelo Hospital das Clínicas e Clínica Dermatológica de Strasbourg, França.
} 
machucados em geral, arranhões e até mesmo, porém de forma menos frequente, acnes inflamadas ${ }^{9}$.

Independentemente do tipo de porta de entrada para a infecção, os sintomas são os mesmos: vermelhidão, inchaço e elevação de temperatura local ou febre. O problema também pode levar à formação de grande coleção purulenta e, em caso mais avançado, absceder de forma espontânea.

A avaliação clínica do caso é de fundamental importância para o correto diagnóstico. Em casos de maiores proporções, é necessário analisar os sinais vitais do paciente, como temperatura corporal, pressão arterial, frequência cardíaca e respiratória. A inspeção e a palpação na região são fundamentais, assim como a avaliação da eventual presença de trismo, dispneia, sialorreia, disfagia e disfonia. Associados a isso, estão os exames por imagem para avaliação do complexo maxilomandibular, a localização do agente causador da infecção e o planejamento da eventual cirurgia ${ }^{10}$. E, também, os exames laboratoriais são importantes auxiliares para a melhor conduta, devendo sempre estar disponível o hemograma para análise geral da série vermelha e branca ${ }^{11}$.

O tratamento típico utilizado para casos de celulite facial é a antibióticoterapia, associada à terapia com calor, além da eliminação do foco de infecção e eventual incisão e drenagem na área afetada. Além disso, em casos mais graves, a internação é necessária, além de tratamento com corticosteroides ${ }^{6}$.

A prevenção é por meio de cuidados básicos de higiene, tanto intrabucais como extraorais. Para isso, a consulta rotineira ao cirurgião-dentista se faz imprescindível, além de atenção a qualquer tipo de alteração tecidual. Também, o ato de apertar espinhas é risco potencial para originar a celulite facial. Dessa forma, a interação bem ajustada entre profissionais da área da saúde, como o cirurgião-dentista e o médico, é fundamental para o adequado desfecho do caso, já que pode haver variação por parte do paciente em procurar um ou outro profissional.

No presente caso clínico, é apresentada a ocorrência de celulite facial no lábio inferior oriunda, provavelmente, de uma acne inflamada, a qual foi a via de entrada das bactérias para os tecidos subcutâneos.

\section{Relato de caso}

Paciente do gênero masculino, 29 anos de idade, compareceu ao profissional cirurgião-dentista com relato de certo inchaço e desconforto no lábio inferior (Figura 1, T0). No exame clínico, foi observada, na área central da borda inferior do lábio, lesão semelhante a uma espinha infeccionada. Houve relato do paciente de tentativa de expelir uma acne naquela área dias antes. No exame clínico, também foi notado relativo edemaciamento da região central do lábio inferior, de consistência firme e com registro de leve dor ao ser pressionado. O profissional cirurgião-dentista optou pelo encaminhamento ao médico dermatologista, uma vez suspeitada a possibilidade de celulite facial.

Sob análise do médico dermatologista, no mesmo T0, foram solicitados exames laboratoriais (Hemograma, Antiestreptolisina O e Proteína C Reativa) e iniciada antibióticoterapia imediata com Cefalexina 500mg, de 8 em 8 horas, por 10 dias, sendo solicitado retorno após 3 dias.

A evolução nos 3 dias subsequentes foi de abrupta piora do quadro clínico (Figura 1, T1 e T3), com piora na capacidade de fala e maior sensibilidade dolorosa ao tato. Na consulta de retorno, foi realizado novo exame clínico, mas optou-se em manter a mesma terapia, acrescentando o uso de um regenerador labial a base de dexpantenol e vitamina $\mathrm{E}$, a fim de evitar ressecamento labial.

No quarto dia após T0 (Figura 1, T4), o caso apresentou-se em seu pico de evolução. O lábio inferior estava altamente edemaciado e desconfigurado, já com áreas de nítidas formações purulentas intradérmicas. A sensibilidade dolorosa ao tato era alta, com queixa de dificuldade da fala e em se alimentar. Apesar de o lábio inferior ainda estar todo inchado, notou-se o início de certa concentração purulenta intradérmica, aparentando uma organização do processo infeccioso.

$\mathrm{Na}$ transição entre o quarto para o quinto dia após T0, tendo sido feita, frequentemente, a terapia com calor, notou-se dois pontos de drenagem, um na parte superior do lábio inferior (local de aparente concentração purulenta intradérmica) e outro na parte central inferior do lábio inferior (local referente à espinha inflamada, provável porta de entrada da infecção) (Figura 1, T5). O paciente relatou que, no momento da compressa térmica, e sob leve pressão, havia expulsão bastante evidente de coleção purulenta com sangue.

Foi mantida a mesma terapia medicamentosa e, nos 3 dias subsequentes (Figura 1, T6, T7 e T8), foi observada gradativa melhora. $\mathrm{O}$ antibiótico foi mantido até completar os 10 dias estipulados inicialmente. A total e completa melhora do quadro, com ausência total de sensibilidade dolorosa e volta da consistência normal do lábio inferior, foi registrada após 30 dias de T0.

Os resultados dos exames solicitados foram normais para o Hemograma e para a Antiestreptolisina O, no entanto, para a Proteína C Reativa o resultado deu alterado, o que evidencia a presença de infecção no organismo, porém de origem diferente do grupo dos estreptococos (Tabela 1). 


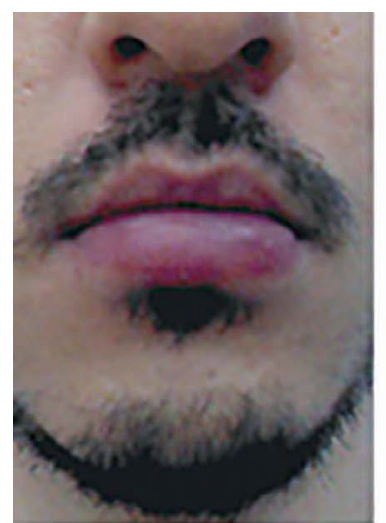

TO

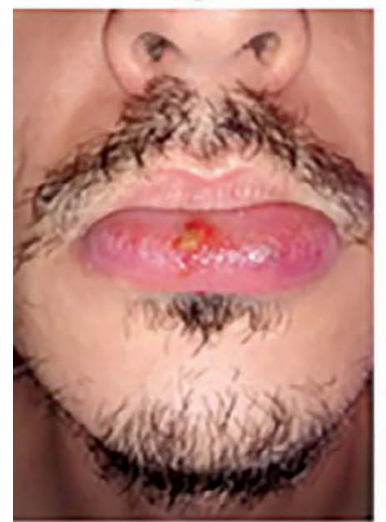

TS: 5 dias após T0

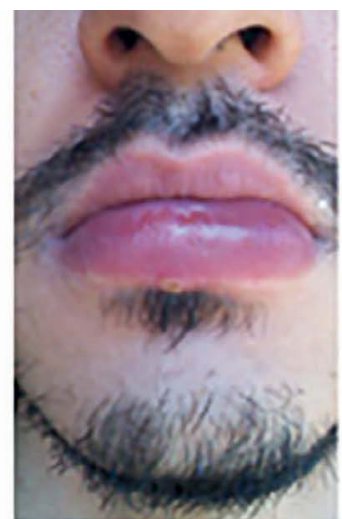

T1: 1 dia após T0

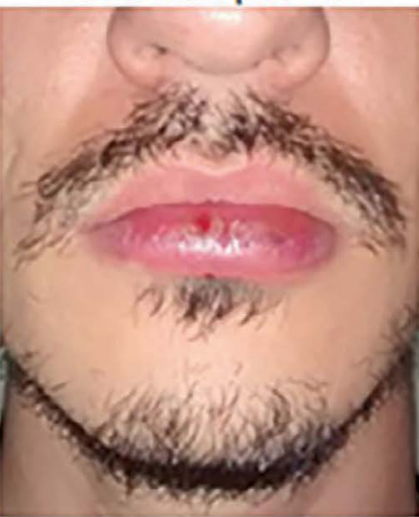

T6: 6 dias após T0

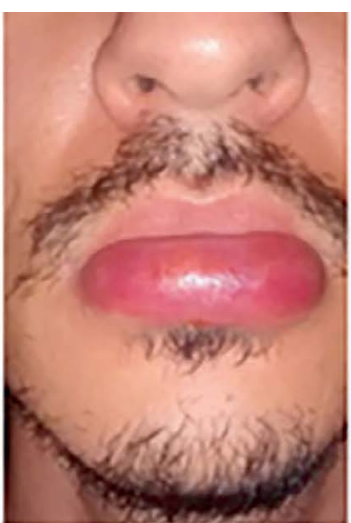

T3: 3 dias após T0

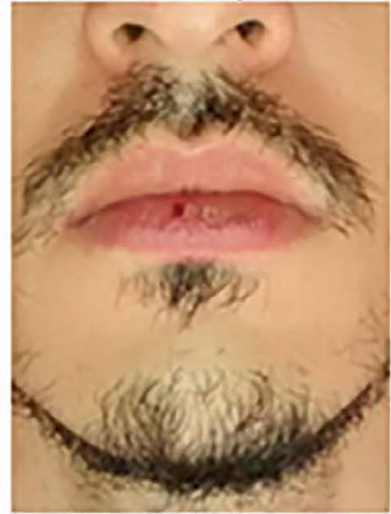

T7: 7 dias após T0

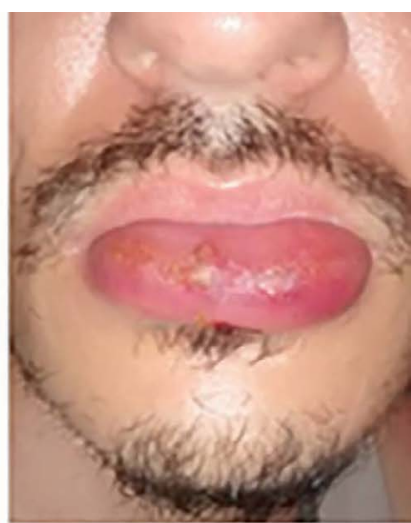

T4: 4 dias após T0

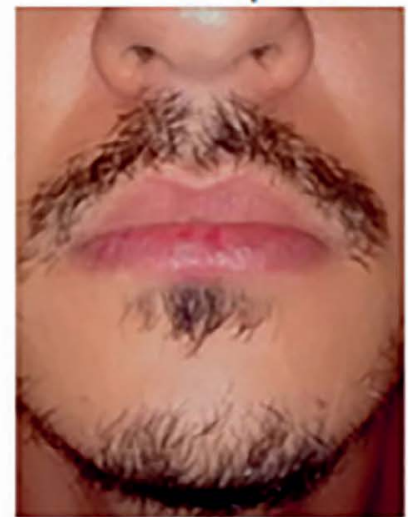

T8: 8 dias após T0

T0 a T8, sendo T0 (procura ao profissional e aspecto inicial), T1 a T3 (3 dias subsequentes a T0 com abrupta piora), T4 (pico de evolução, 4 dias após T0), T5 (ponto de drenagem, 5 dias após T0), T6 a T8 (gradativa melhora com expulsão da coleção purulenta e retardo do inchaço, de 6 até 8 dias após T0).

Figura 1 - Evolução clínica geral do caso

Tabela 1 - Exames solicitados, valores de referência e resultados obtidos

\begin{tabular}{|c|c|c|c|}
\hline \multicolumn{2}{|c|}{ Exames } & Valor de referência & Resultado \\
\hline \multicolumn{2}{|c|}{$\begin{array}{l}\text { Antiestreptolisina O } \\
\text { Proteína C Reativa }\end{array}$} & $\begin{array}{l}\text { Até } 200 \mathrm{UI} / \mathrm{mL} \\
\text { Até } 6 \mathrm{mg} / \mathrm{L}\end{array}$ & $\begin{array}{l}32 \mathrm{UI} / \mathrm{mL} \\
22.8 \mathrm{mg} / \mathrm{L}\end{array}$ \\
\hline \multirow{16}{*}{ Hemograma } & Eritrócitos & 4.5 a 5.5 milhões $/ \mathrm{mm}^{3}$ & 4.95 milhões $/ \mathrm{mm}^{3}$ \\
\hline & Hemoglobina & 14 a $18 \mathrm{~g} / \mathrm{dl}$ & $14.6 \mathrm{~g} / \mathrm{dl}$ \\
\hline & Vol. Globular & 40 a $52 \%$ & $44.4 \%$ \\
\hline & VGM & 82 a $92 n$ & $89.7 n$ \\
\hline & HGM & 27 a 31 pg & $29.5 \mathrm{pg}$ \\
\hline & $\mathrm{CHGM}$ & 32 a 36 g/dl & $32.9 \mathrm{~g} / \mathrm{dl}$ \\
\hline & $\mathrm{RDW}-\mathrm{CV}$ & 11 a $15 \%$ & $12.4 \%$ \\
\hline & Leucócitos & 4.300 a $10.000 / \mathrm{mm}^{3}$ & $4.850 / \mathrm{mm}^{3}$ \\
\hline & Neutrófilos & 1.076 a $7.300 / \mathrm{mm}^{3}$ & $2.842 / \mathrm{mm}^{3}$ \\
\hline & Eosinófilos & 60 a $700 / \mathrm{mm}^{3}$ & $39 / \mathrm{mm}^{3}$ \\
\hline & Basófilos & 0 a $200 / \mathrm{mm}^{3}$ & $29 / \mathrm{mm} 3$ \\
\hline & Monócitos & 86 a $1.200 / \mathrm{mm}^{3}$ & $558 / \mathrm{mm} 3$ \\
\hline & Linfócitos & 1.000 a $5.2000 / \mathrm{mm}^{3}$ & $1.382 / \mathrm{mm}^{3}$ \\
\hline & Linfócitos atípicos & $0 / \mathrm{mm}^{3}$ & $0 / \mathrm{mm}^{3}$ \\
\hline & Plasmócitos & $0 / \mathrm{mm}^{3}$ & $0 / \mathrm{mm}^{3}$ \\
\hline & Plaquetas & 120.000 a $420.000 / \mathrm{mm}^{3}$ & $271.000 / \mathrm{mm}^{3}$ \\
\hline
\end{tabular}




\section{Discussão}

As celulites faciais são infecções graves de características agudas, com rápida progressão (2 a 4 dias), localização difusa, ausência de secreção purulenta, em alguns casos, consistência endurecida, área hiperêmica e causada por bactérias mistas, apresentando potencial risco à saúde do indivíduo ${ }^{1}$.

Diferentes trabalhos já apresentaram situações de infecções graves de origem dentária, que culminaram em infecções graves. Uma vez constatada origem odontogênica e abscesso dentoalveolar, deve-se tentar, inicialmente, a preservação do elemento dental e adequação do meio bucal, mas, quando não for possível, a exodontia do elemento envolvido deve ser realizada. A eliminação do fator causador permite melhora dos sinais e sintomas do quadro de infecção, e a drenagem da coleção purulenta também é recomendada ${ }^{1,9}$.

Porém, quando se trata de celulite facial sem origem dentária, como foi o caso relatado na presente pesquisa, adequação do meio e extrações não se justificam. Como relatado, outras vias de infecção são totalmente possíveis, e a acne ou espinha infeccionada pode gerar uma infecção local que se dissemina nos planos subcutâneos.

Quando tal fato ocorre em área de tecido mole vascular frouxo, tal como no lábio inferior, o edema se torna bastante evidente devido à maior flacidez do local, o que gera uma evolução extremamente rápida do caso e consequente incômodo ao paciente.

Caso haja geração de abscesso e drenagem espontânea, estando o paciente coberto por terapia medicamentosa, a resolução do caso, geralmente, é feita de forma mais acelerada, tal como ocorreu no presente caso. Os exames laboratoriais também são capazes de nortear a decisão do profissional por qual conduta tomar. Por isso, exames como Hemograma, Proteína C Reativa (substância presente no sangue que indica inflamação ou infecção no organismo) e Antiestreptolisina O (indicador de infecções estreptocócicas) são fundamentais para a correta conduta do caso. No relato em questão, em que este último exame não apresentou alterações, imagina-se que a infecção teria origem microbiológica por estafilococos (Tabela 1). Por isso, é importante também que se opte por tratamento com antibióticos de largo espectro.

Azenha et al. ${ }^{1}$ (2012) relatam que a antibióticoterapia é uma grande aliada no tratamento das infecções faciais, porém, tem papel coadjuvante no tratamento, pois deve sempre ser associada à drenagem e à remoção da causa. Os autores ainda afirmam que a simples administração não causa efeito algum, podendo, em alguns casos, exacerbar e intensificar a gravidade da infecção após o período de administração.

No entanto, é notório que a afirmação desses autores se justifica em casos de celulite facial com origem etiológica definida, como a odontogênica, em que há um agente causal específico. Entretanto, casos como o relatado no presente artigo, em que houve uma via de entrada definida (acne inflamada), porém, não há agente etiológico possível de ser removido, é indicada, sim, apenas a correta higienização acompanhada da antibióticoterapia, que pode ser bem sucedida, como citado por Calzadilla ${ }^{12}$ (1997). O acompanhamento da evolução do caso de forma criteriosa e cautelosa é também imprescindível.

Fato é que o profissional envolvido deve estar atento à evolução do caso, munido de todos os exames necessários e informado da forma mais viável e eficaz para tratamento do caso. Além disso, é de suma importância a interdisciplinaridade entre os profissionais, tanto da área odontológica quanto da médica, já que, muitas vezes, o paciente procura pelo profissional que lhe gera maior confiança, mas não necessariamente sua área de atuação será a mais adequada para resolução do caso.

\section{Conclusões}

As celulites faciais são infecções graves que, se não corretamente tratadas, podem gerar complicações indesejadas. Em casos de origem definida, em que há agente causal determinado, faz-se necessária sua remoção, associada à drenagem e à antibióticoterapia. Já em casos como o relatado neste trabalho, em que não há fator etiológico a ser removido, o acompanhamento associado à antibióticoterapia é fundamental, junto de meticulosa higienização da área afetada. É importante ressaltar que, além do correto exame clínico, é fundamental o diagnóstico por meio de exames de imagem e/ou exames laboratoriais, para uma correta análise microbiológica e um tratamento direcionado.

\section{Abstract}

Introduction: facial cellulitis is the diffusion of inflammatory process by subcutaneous planes in soft tissues; its etiology potentially comes from several factors and the odontogenic origin is the one most commonly found. However, facial cellulitis may also be related to less common causes, such as infectious processes from non-odontogenic origin. Objective: the present case report clarifies the beginning of an infectious process generated by inflamed acne lesions on the lower lip border, which was the gateway to bacteria, producing the diffusion of inflammatory process to subcutaneous planes and originating facial cellulitis. Case report: because there is no specific causal agent such as those produced by odontogenic causes, the treatment with broad-spectrum antibiotic therapy has been established, associated with meticulous hygiene and heat therapy to induce spontaneous abscessation. Specific laboratory tests were also requested for higher safety and accurate diagnosis of the professional. The evolution of the case 
occurred in the course of 8 days, quickly and acutely, especially in the first 5 days. Final considerations: as reported in the case, the intimate interaction between the dentist and the physician, and the presence of interdisciplinarity were fundamental factors in the treatment as a whole to solve the case satisfactorily, considering the occurrence of facial cellulitis in such areas are not usually witnessed.

Keywords: Facial cellulitis. Drainage. Infection. Lower lip.

\section{Referências}

1. Azenha MR, de Lacerda AS, Bim AL, Caliente R, Guzman S. Celulite facial de origem odontogênica. Apresentação de 5 casos. Rev Cir Traumatol Buco-Maxilo-Fac Camaragibe 2012; 12(3):41-8.

2. Calzadilla O. Diagnóstico y tratamiento de la celulitis facial odontódena. Act Odont Venez 2001; 39:1-10.

3. Dice W, Pryor EJ, Kilpatrick W. Facial cellulitis following dental injury in child. Ann Emerg Med 1982; 11:541-5.

4. Schusterman S. Pediatric dental update. Pediatr Rev 1994; 15:311-8.

5. Hodges T, Cohen DA, Deck D. Odontogenic sinus tracts. Am Fam Psysician 1989; 40:113-6.

6. Itiere Odzili FA, Mahoungou Guimbi K, Boumandoki PJ, Otiobanda GF, Ovoundard M, Ondzotto G. 67 cases of face and neck cellulitis managed at the Brazzaville Teaching Hospital. Rev Stomatol Chir Maxillofac Chir Orale 2014; 115(6):349-52.

7. Allen CM, Bouquot JE, Damm DD, Neville BW. Patologia oral e maxilofacial. Rio de Janeiro: Guanabara Koogan; 1998.

8. Almeida AM, Lia RCC. Disseminação de infecção purulenta envolvendo segundo e terceiro molares inferiores - relato de caso clínico. Rev Brasileira de Cirurgia e Implantodontia 2000; 7:34-6.

9. Brito N, Rodrigues F, Salgado M, Lemos L. Celulite facial de provável causa odontogênica. Saúde Infantil 2005; 27(2):55-7.

10. Vasconcelos BEC, Cauás M, Albert DGM, Nascimento GJF, Holanda GZ. Disseminação de infecção odontogênica através das fáscias cervicais profundas: relato de caso clínico. Rev Cir Traumatol Buco-Maxilo-Fac Camaragibe 2002; 2:21-5.

11. Carvalho ACP, Carvalho PSP, Andrade ED, Passeri LA. Tratamento dos abscessos de origem dental. Odontol Mod 1988; 15:34-9.

12. Calzadilla O. Celulitis facial odontógena. Rev Cub Estomatol 1997; 34:15-20.

\section{Endereço para correspondência:}

Marcelo Bighetti Toniollo

Rua Primo Tronco, 81 - apto 23

14030-020 Vila Virgínea, Ribeirão Preto

Fone: (64)9-9922-7518

E-mail: martoniollo@yahoo.com.br

Recebido: 29/09/2016. Aceito: 24/03/2017. 